\title{
The Measurement of the Seaquark Sivers Asymmetry in Fermilab E1039 Polarized Drell-Yan Experiment
}

LANL : M. Yurov

E1039 SeaQuest collaboration

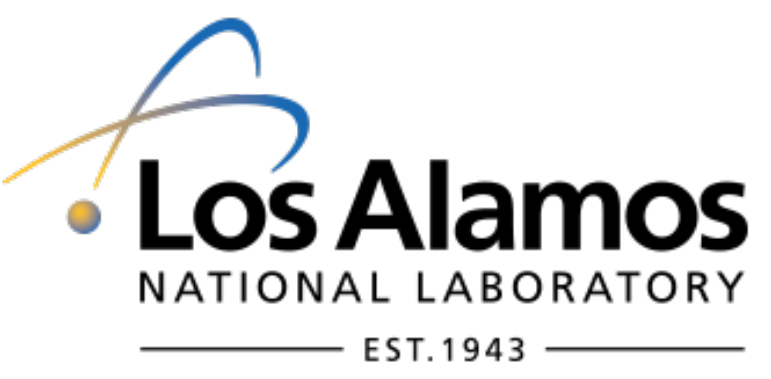

5th Joint Meeting of the APS DNP and the Physical Society of Japan 10/18, Waikoloa, HI 


\section{WHY ARE WE DOING E1039?}

$$
\frac{1}{2}=\frac{1}{2} \Delta \Sigma+L_{q}+L_{\bar{q}}+\Delta G+L_{g}
$$

need for a major breakthrough in understanding of the nucleon spin composition

importance of sea quarks

account for spin of quarks and gluons still missing $\sim$ half the picture

sea quark OAM could be a major part of missing spin 


\section{HINTS OF NON-ZERO OAM}

sea quarks flavor asymmetry observation - enhanced $\frac{\bar{d}}{\bar{u}}$ in E866

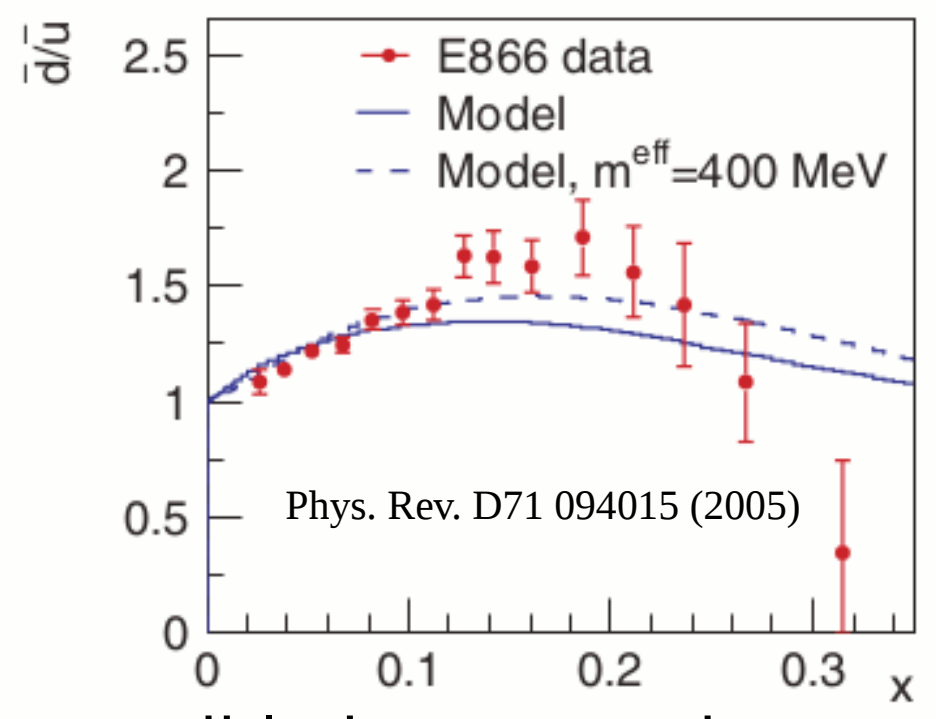

pion cloud model as possible interpretation

$$
|p\rangle \propto\left|p_{0}\right\rangle+\left|n \pi^{+}\right\rangle+\left|\Delta^{++} \pi^{-}\right\rangle+\ldots
$$

needs to conserve parity: pions negative parity requires $L=1$

if correct description - $|u \bar{d}\rangle$ possess OAM

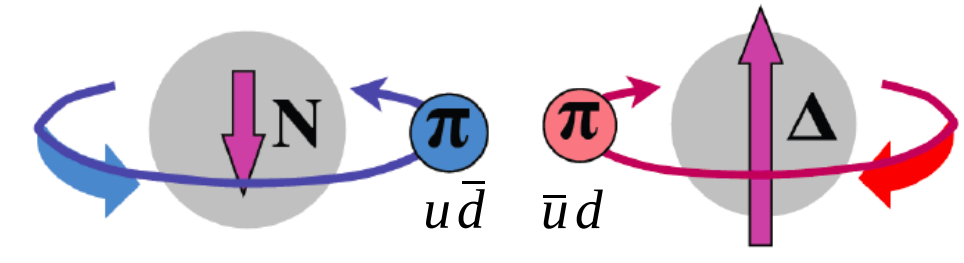

E866 results might point to sea quarks OAM 


\section{SIVERS FUNCTION}

explore correlation of quark motion relative to nucleon spin direction

eight leading order Transverse Momentum Distributions (TMDs)

Sivers function - transverse momentum distribution of unpolarized quarks in a transversely polarized proton

$f \frac{\perp}{1 T}\left(x, k_{T}\right)=\bigodot-\bigodot$

proposed to explain results of experiments such as SSA in $p p^{\uparrow} \rightarrow \pi X$ (E704)

points to possible intrinsic $k_{T}$ imbalance leads to asymmetry

Sivers function vanishes if quarks have no OAM!

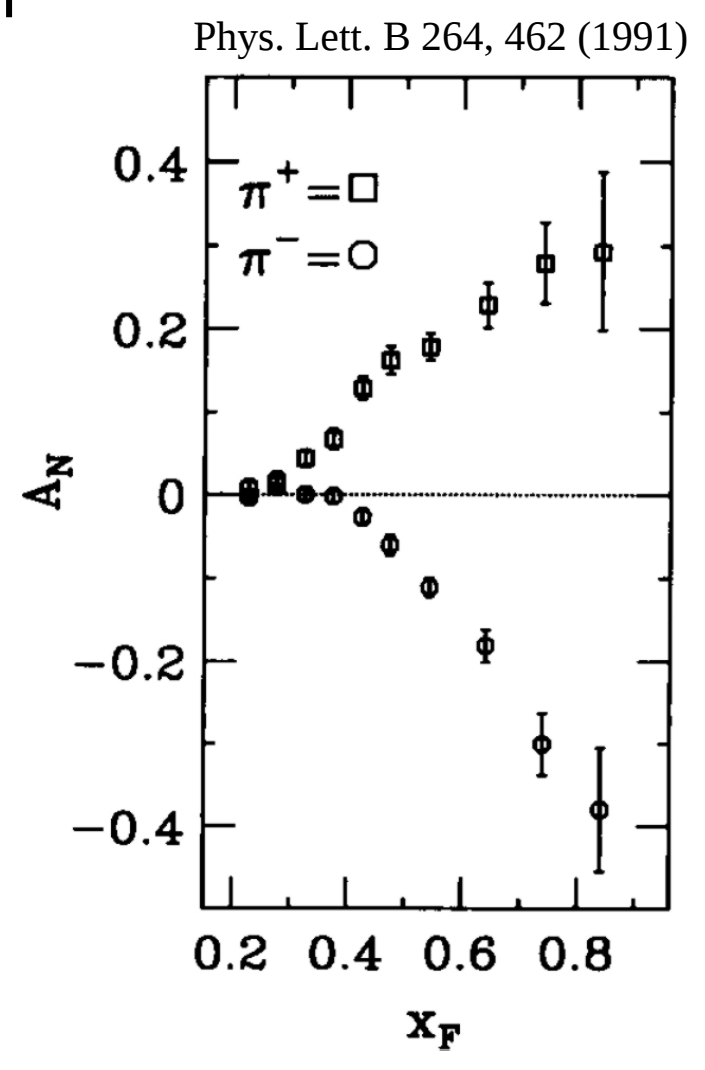




\section{ACCESSING SEA QUARK SIVERS FUNCTION}

$$
e+p^{\uparrow} \rightarrow e^{\prime} \pi X
$$

(1) Polarized Semi-Inclusive DIS

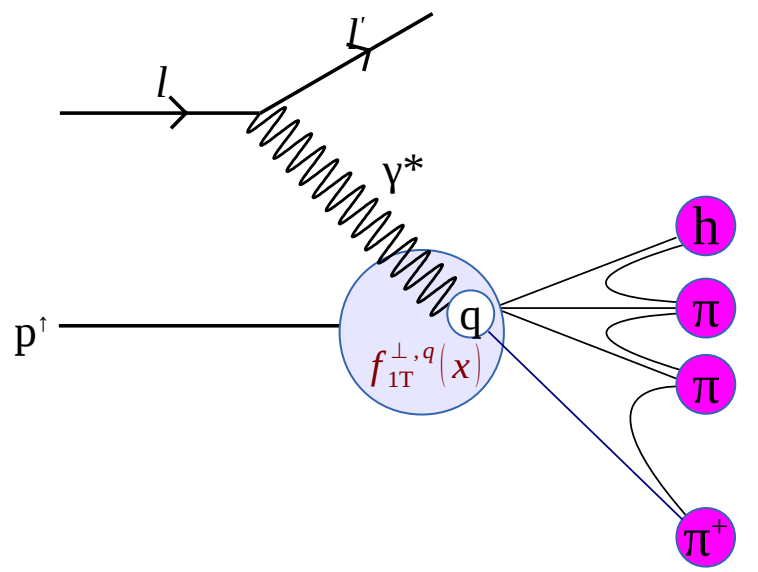

$A_{U T}^{S I D I S} \propto \frac{\sum_{q} e_{q}^{2} f_{1 T}^{\perp, q}(x) \otimes D_{1}^{q}(Z)}{\sum_{q} e_{q}^{2} f_{1}^{q}(x) \otimes D_{1}^{q}(Z)}$

- L-R asymmetry in hadron production

- quark to hadron fragmentation function

- valence-sea quark: mixed

$$
p+p^{\uparrow} \rightarrow \mu^{+} \mu^{-} X
$$

(2) Polarized Drell-Yan

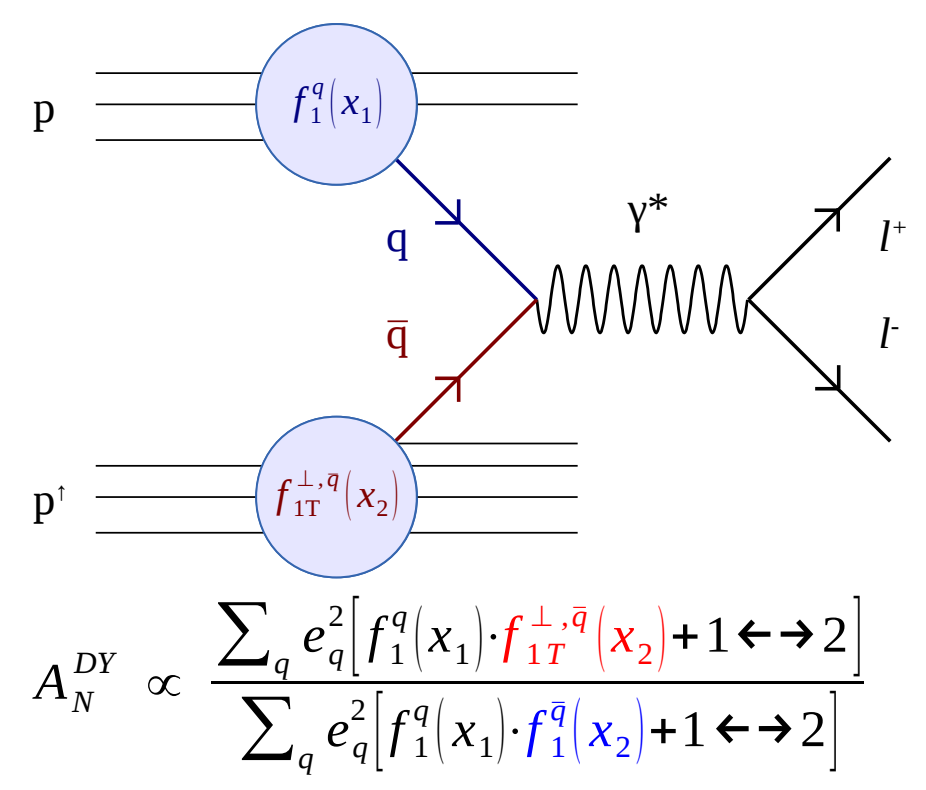

- L-R asymmetry in Drell-Yan production

- no fragmentation function

- valence-sea quark: isolated 


\section{ACCESSING SEA QUARK SIVERS FUNCTION}

$$
e+p^{\uparrow} \rightarrow e^{\prime} \pi X
$$

(1) Polarized Semi-Inclusive DIS

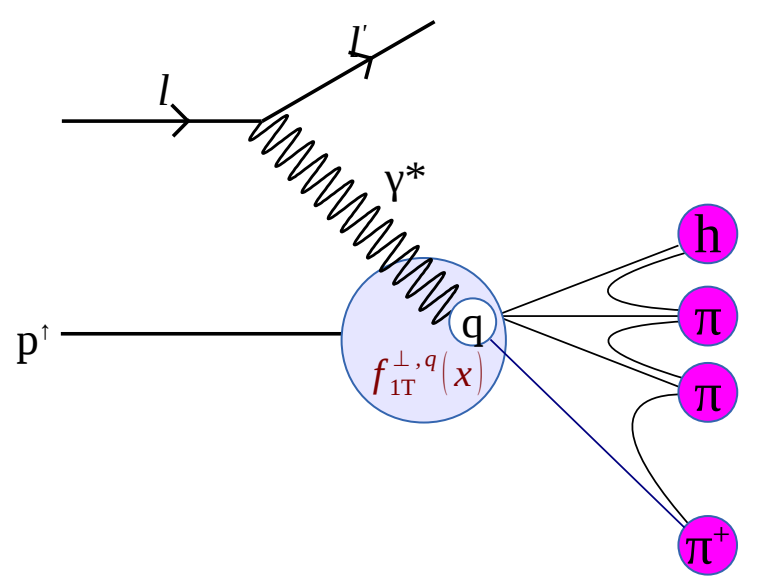

$A_{U T}^{\text {SIDIS }} \propto \frac{\sum_{q} e_{q}^{2} f_{1 T}^{\perp, q}(x) \otimes D_{1}^{q}(z)}{\sum_{q} e_{q}^{2} f_{1}^{q}(x) \otimes D_{1}^{q}(z)}$

- L-R asymmetry in hadron production

- quark to hadron fragmentation function

- valence-sea quark: mixed

$$
p+p^{\uparrow} \rightarrow \mu^{+} \mu^{-} X
$$

(2) Polarized Drell-Yan

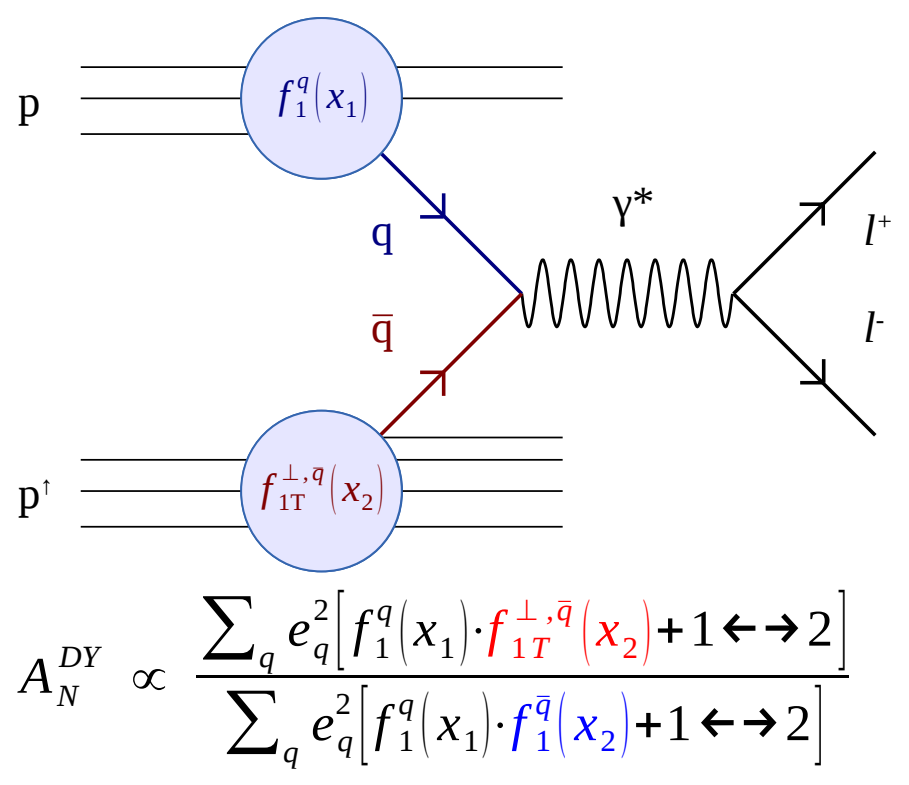

- L-R asymmetry in Drell-Yan production

- no fragmentation function

- valence-sea quark: isolated 


\section{ACCESSING SEA QUARK SIVERS FUNCTION}

has not been tried yet

O only experiment sensitive to sea quarks at large $x$

\begin{tabular}{lcc}
\hline \hline quark & SIDIS & Drell-Yan \\
\hline valence & known & COMPASS \\
\hline \multirow{2}{*}{ sea } & $\begin{array}{c}\text { poor } \\
\text { sensitivity }\end{array}$ & $\begin{array}{c}\text { unknown } \\
\text { E1039 }\end{array}$ \\
\hline \hline
\end{tabular}

selects sea quark from target

$\frac{d^{2} \sigma}{d x_{b} d x_{t}}=\frac{4 \pi \alpha^{2}}{9 x_{b} x_{t}} \frac{1}{s} \sum_{i} e_{i}^{2}$

$$
\times\left\{q_{i}\left(x_{b}\right) \bar{q}_{i}\left(x_{t}\right)+\bar{q}_{i}\left(x_{b}\right) q_{i}\left(x_{t}\right)\right\}
$$

- for E1039 kinematic configuration first term dominates

measure Sivers asymmetry for both

- $\bar{u}(x), \bar{d}(x)$

- determine possible flavor asymmetry

$$
p+p^{\uparrow} \rightarrow \mu^{+} \mu^{-} X
$$

(2) Polarized Drell-Yan

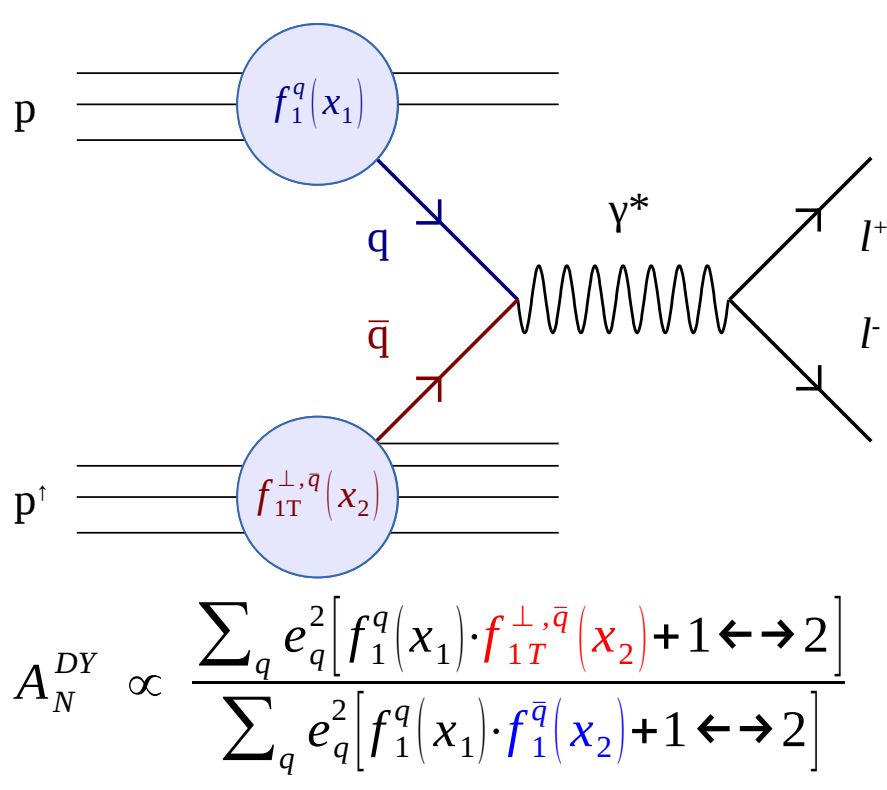

- L-R asymmetry in Drell-Yan production

- no fragmentation function

- valence-sea quark: isolated 


\section{FERMILAB E1039 EXPERIMENT}

FNAL $120 \mathrm{GeV}$ proton beam

- $\sqrt{S}=15.5 \mathrm{GeV}$

- $5 \times 10^{12} \mathrm{p} / \mathrm{spill} ; 5 \mathrm{~s} / \mathrm{min}$

- $7.7 \times 10^{17}$ protons on target/year
SeaQuest E906 spectrometer

- 4 tracking station, trigger hodoscope

- focusing \& analyzing magnets

- iron dump

LANL/UVA polarized target

- solid $\mathrm{NH}_{3}, \mathrm{ND}_{3}$

- $5 \mathrm{~T}$ field, $1 \mathrm{~K}$ fridge

- $\mu$-wave freq. P flip

$\frac{\text { ENAL proton beam }}{E_{\text {beam }}}=120 \mathrm{GeV}=$

$$
\gamma^{*} \rightarrow \mu+\mu-
$$

seaQues

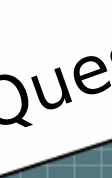

uest $M$

spectrometer nuon Spe
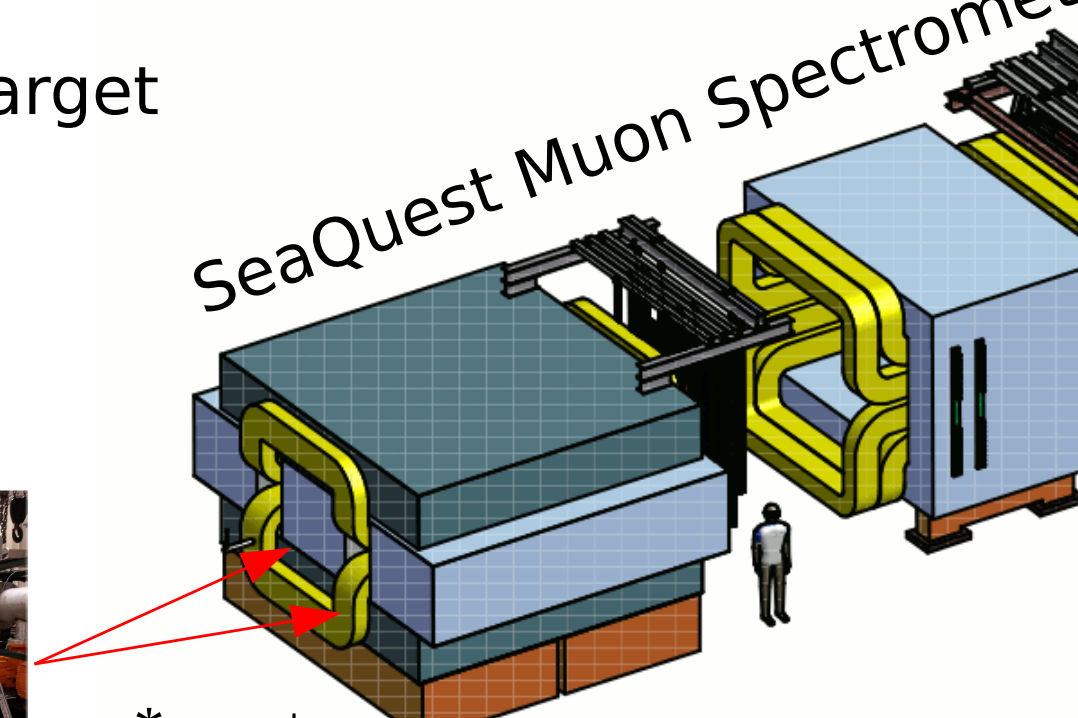

$A_{N}\left(p_{\text {beam }}+p_{\text {trg }}^{\uparrow} \rightarrow \mathrm{DY}\right) \propto \frac{N_{L}^{D Y}-N_{R}^{D Y}}{N_{L}^{D Y}+N_{R}^{D Y}} \propto \frac{f_{1 T}^{\perp, \bar{u}}\left(x_{t}\right)}{f_{1}^{\bar{u}}\left(x_{t}\right)}$

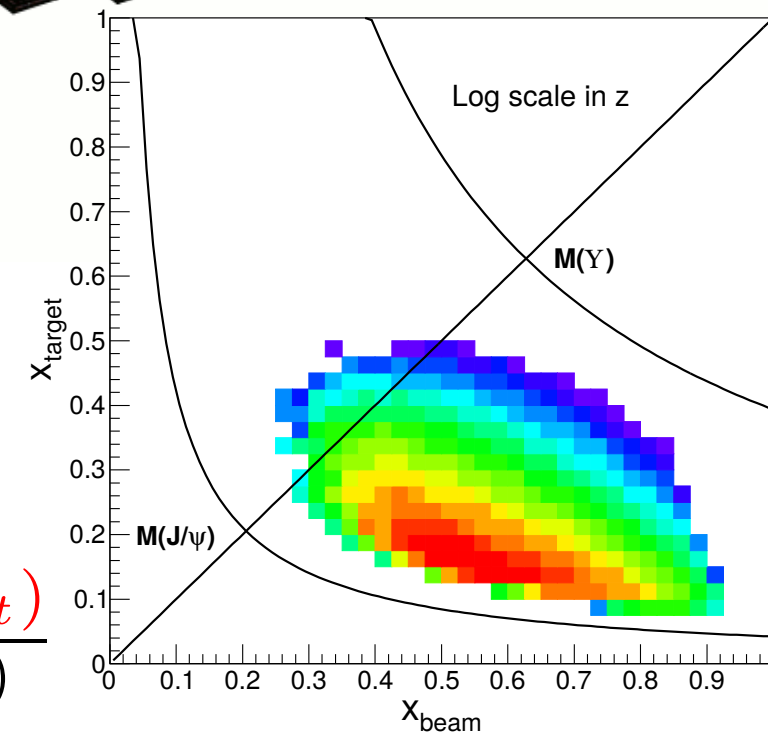

M. Yurov, LANL 


\section{FERMILAB E1039 EXPERIMENT}

asymmetry estimates from proposal (2013)

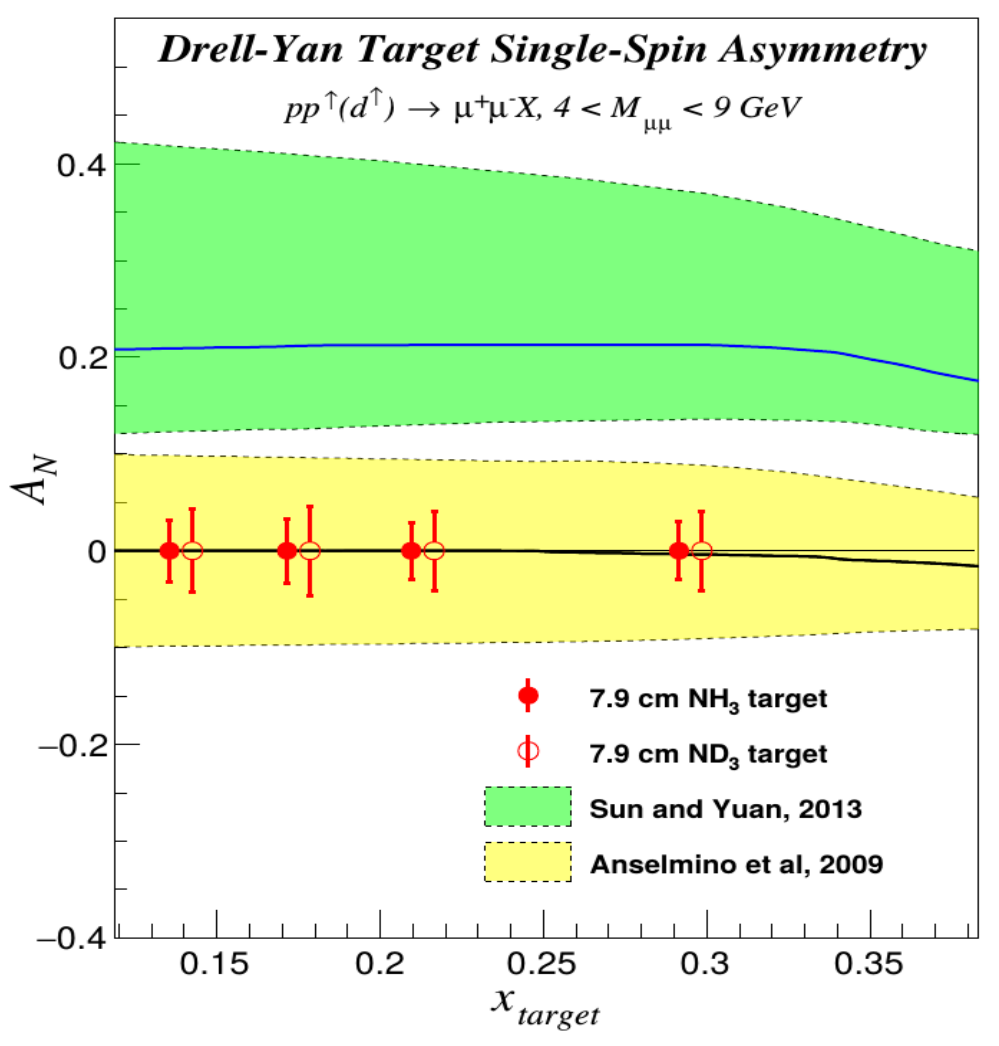

- sign and value of sea quark Sivers asymmetry

- sea quark Sivers flavor dependence

if non-zero, "smoking gun" evidence for sea quark OAM

statistics estimates and kinematics coverage

Range $\mathrm{x}_{2} \quad$ Mean $\mathrm{x}_{2} \quad \mathrm{~N}$ events $\mathrm{p} \quad \Delta \mathrm{A} \% \mathrm{p} \quad \mathrm{N}$ events $\mathrm{n} \quad \Delta \mathrm{A} \% \mathrm{n}$

$\begin{array}{llllll}0.1-0.16 & .139 & 5.0 \times 10^{4} & 3.2 & 5.8 \times 10^{4} & 5.4 \\ 0.16-0.19 & 0.175 & 4.5 \times 10^{4} & 3.3 & 5.2 \times 10^{4} & 5.7 \\ 0.19-0.24 & 0.213 & 5.7 \times 10^{4} & 2.0 & 6.6 \times 10^{4} & 5.0 \\ 0.24-0.6 & 0.295 & 5.5 \times 10^{4} & 3.0 & 6.4 \times 10^{4} & 5.1\end{array}$




\section{FERMILAB E1039 EXPERIMENT}

\section{(Un)Polarized Drell-Yan Experiments}

\begin{tabular}{|c|c|c|c|c|c|c|c|c|}
\hline Experiment & Particles & $\underset{(\mathrm{GeV})}{\text { Energy }}$ & $x_{b}$ or $x_{t}$ & $\underset{\left(\mathrm{cm}^{-2} \mathrm{~s}^{-1}\right)}{\text { Luminosity }}$ & & $P_{b}$ or $P_{t}(f)$ & rFOM & Timeline \\
\hline $\begin{array}{l}\text { COMPASS } \\
\text { (CERN) }\end{array}$ & $\pi^{ \pm}+\mathbf{p}^{\dagger}$ & $\begin{array}{l}160 \mathrm{GeV} \\
\sqrt{\mathrm{s}}=17\end{array}$ & $x_{t}=0.2-0.3$ & $2 \times 10^{33}$ & 0.14 & $\begin{array}{c}P_{t}=90 \% \\
f=0.22\end{array}$ & $1.1 \times 10^{-3}$ & 2014,2018 \\
\hline $\begin{array}{l}\text { PANDA } \\
\text { (GSI) }\end{array}$ & $\mathbf{p}+\mathbf{p}^{\dagger}$ & $\begin{array}{l}15 \mathrm{GeV} \\
\sqrt{\mathrm{s}}=5.5\end{array}$ & $x_{t}=0.2-0.4$ & $2 \times 10^{32}$ & 0.07 & $\begin{array}{c}P_{t}=90 \% \\
f=0.22\end{array}$ & $1.1 \times 10^{-4}$ & $>2018$ \\
\hline $\begin{array}{l}\text { PAX } \\
\text { (GSI) }\end{array}$ & $\mathbf{p}^{\dagger}+\mathbf{p}$ & $\begin{array}{l}\text { collider } \\
\sqrt{s}=14\end{array}$ & $x_{b}=0.1-0.9$ & $2 \times 10^{30}$ & 0.06 & $P_{b}=90 \%$ & $2.3 \times 10^{-5}$ & $>2020 ?$ \\
\hline $\begin{array}{l}\text { NICA } \\
\text { (JINR) }\end{array}$ & $\mathbf{p}^{\dagger}+\mathbf{p}$ & $\begin{array}{l}\text { collider } \\
\sqrt{s}=26\end{array}$ & $x_{b}=0.1-0.8$ & $1 \times 10^{31}$ & 0.04 & $P_{b}=70 \%$ & $6.8 \times 10^{-5}$ & $>2018$ \\
\hline $\begin{array}{l}\text { PHENIX } \\
\text { (RHIC) }\end{array}$ & $\mathbf{p}^{\dagger}+\mathbf{p}$ & $\begin{array}{l}\text { collider } \\
\sqrt{s}=500\end{array}$ & $x_{b}=0.05-0.1$ & $2 \times 10^{32}$ & 0.06 & $P_{b}=60 \%$ & $3.6 \times 10^{-4}$ & $>2018$ \\
\hline $\begin{array}{l}\text { SeaQuest } \\
\text { (FNAL: E-906) }\end{array}$ & $p+p$ & $\begin{array}{l}120 \mathrm{GeV} \\
\sqrt{\mathrm{s}}=15\end{array}$ & $\begin{array}{l}x_{b}=0.35-0.9 \\
x_{t}=0.1-0.45\end{array}$ & $3.4 \times 10^{35}$ & --- & --- & -- & $2012-2015$ \\
\hline $\begin{array}{l}\text { Pol tgt DY } \\
\text { (FNAL: E-1039) }\end{array}$ & $\mathbf{p}+\mathbf{p}$ & $\begin{array}{l}120 \mathrm{GeV} \\
\sqrt{\mathrm{s}}=15\end{array}$ & $x_{t}=0.1-0.45$ & $4.4 \times 10^{35}$ & $\begin{array}{l}0- \\
0.2^{*}\end{array}$ & $\begin{array}{c}P_{t}=80 \% \\
f=0.176\end{array}$ & 0.13 & 2016 \\
\hline \multirow[t]{2}{*}{$\begin{array}{l}\text { Pol beam DY } \\
\text { (FNAL: E-1027) }\end{array}$} & $\mathbf{p}^{\dagger}+\mathbf{p}$ & $\begin{array}{l}120 \mathrm{GeV} \\
\sqrt{\mathrm{s}}=15\end{array}$ & $x_{b}=0.35-0.9$ & $2 \times 10^{35}$ & 0.04 & $P_{b}=60 \%$ & 1 & 2018 \\
\hline & \multicolumn{8}{|c|}{$\begin{array}{l}\begin{array}{l}\ddagger 8 \mathrm{~cm} \mathrm{NH} \\
\text { target }\end{array}{ }^{5} \mathrm{~L}=1 \times 10^{36} \mathrm{~cm}^{-2} \mathrm{~s}^{-1}\left(\mathrm{LH}_{2} \text { tgt limited }\right) / \mathrm{L}=2 \times 10^{35} \mathrm{~cm}^{-2} \mathrm{~s}^{-1}(10 \% \text { of Ml beam limited }) \\
{ }^{*} \text { not constrained by SIDIS data } /{ }^{*} \mathrm{rFOM}=\text { relative lumi * } \mathrm{P}^{2}{ }^{*} \mathrm{f}^{2} \text { wrt } \mathrm{E}-1027 \text { ( } \mathrm{f}=1 \text { for pol p beams) }\end{array}$} \\
\hline
\end{tabular}

W. Lorenzon (U-Michigan) 
The experiment is fully funded by DOE: stage-2 approval May, 2018

polarized target ( $>90 \%$ of $p$ polarization)

- rebuild superconducting magnet, rotate coils

- modify $1 \mathrm{~K}$ evaporative refrigerator

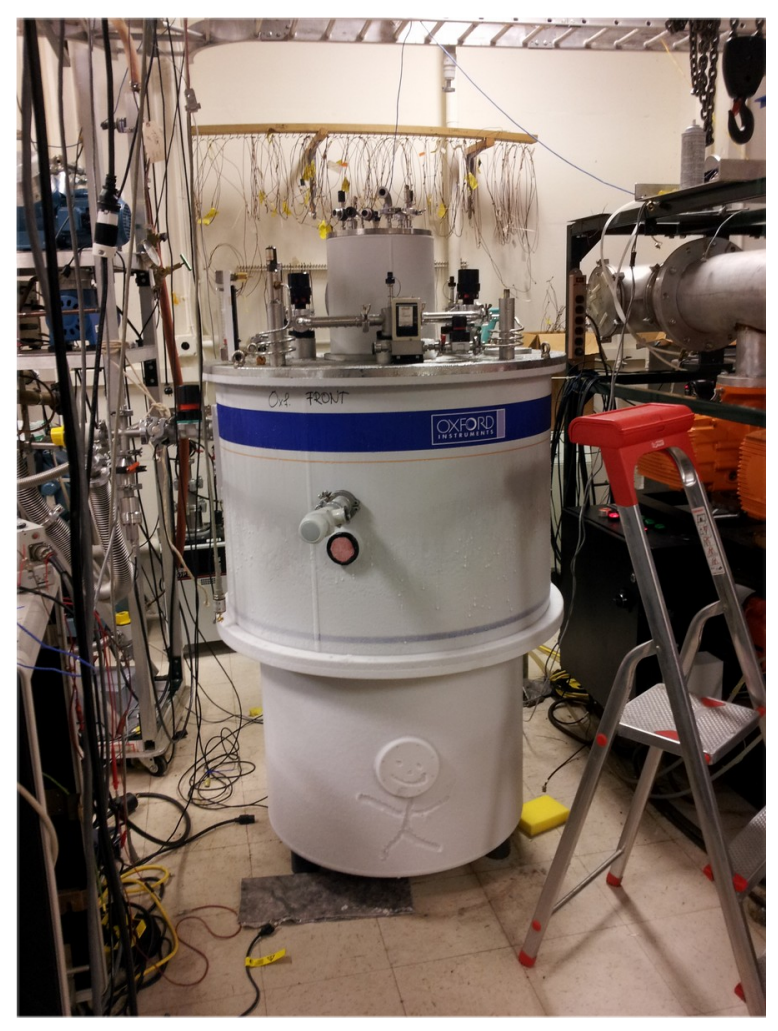


The experiment is fully funded by DOE: stage-2 approval May, 2018

polarized target ( $>90 \%$ of p polarization)

- rebuild superconducting magnet, rotate coils

- modify $1 \mathrm{~K}$ evaporative refrigerator
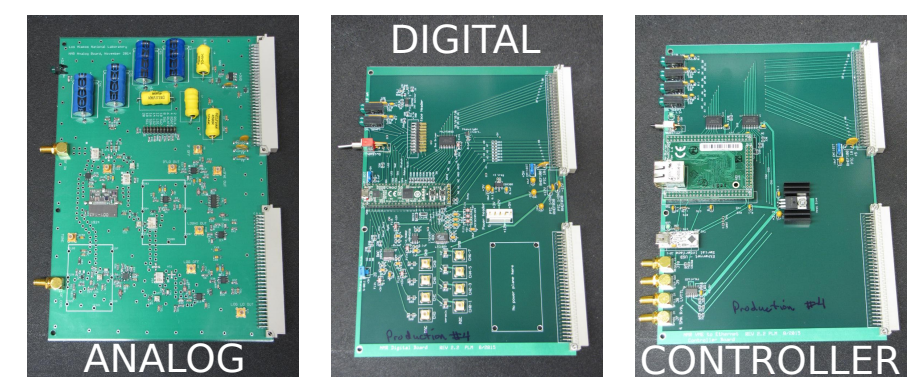

new NMR system

- replacing 30 years-old Liverpool Q-meter

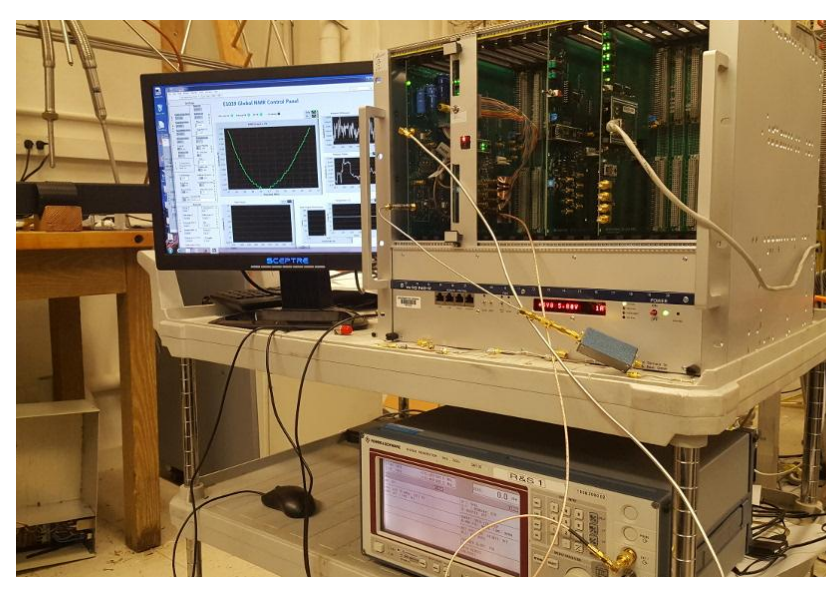


The experiment is fully funded by DOE: stage- 2 approval May, 2018

polarized target ( $>90 \%$ of $p$ polarization)

- rebuild superconducting magnet, rotate coils

- modify $1 \mathrm{~K}$ evaporative refrigerator

new NMR system

- replacing 30 years-old Liverpool Q-meter

LHe liquefier for helium recirculation

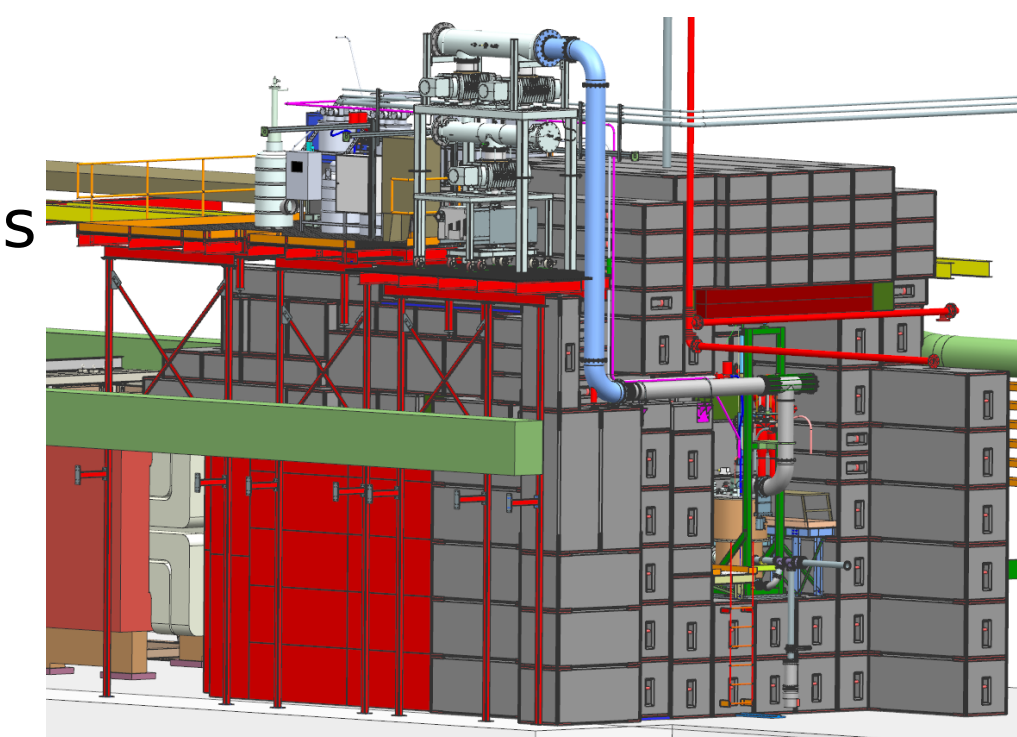

major modifications in Experimental Hall

- new shielding design, beam collimators upstream

- move target $200 \mathrm{~cm}$ upstream, target cave, cryo-platform

inherit spectrometer from SeaQuest E906

- dedicated trigger, online vertex reconstruction (res. $30 \mathrm{~cm}$ ) 
Current timeline:

May, $2018 \bigcirc$ E906 decommissioning

"Today" = Oct, $2018 \bigcirc$ installation is in progress

Nov, $2018 \bigcirc$ collaboration meeting

Nov, $2018 \bigcirc$ last UVA target cooldown

Dec, $2018 \bigcirc$ shielding, target cave, cryo-platform

Jan, $2019 \bigcirc$ target is shipped to FNAL, target installation

March, 2019

May, 2019

2019-2021

E1039 commissioning starts

LHe liquefier installation

E1039 approved to run 2 years 


\section{E1039 COLLABORATION}

\section{We are inviting you to E1039 collaboration}

expertise, manpower, ...

Contact Spokespersons: Kun Liu (liuk@fnal.gov) - LANL Dustin Keller (dustin@jlab.org) - UVA

Please talk to me after the talk: myurov@lanl.gov

Learn more about E1039:

https://wiki.shanti.virginia.edu/display/SeaQuest/ 
THANK YOU

M. Yurov, LANL 


\section{RECENT SIVERS EXTRACTION}
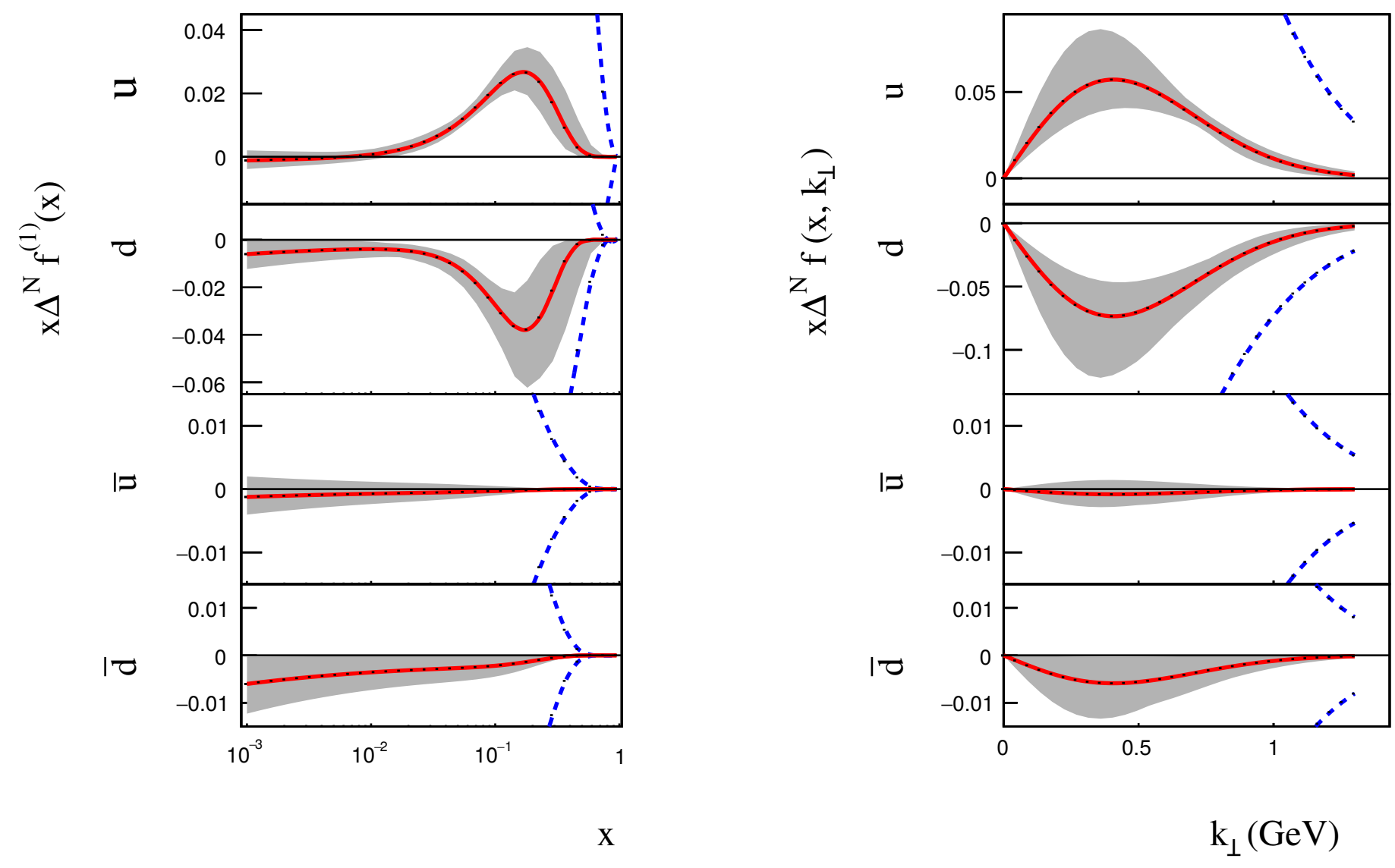

Anselmino, M. et. all, https://doi.org/10.1007/JHEP04(2017)046 